\title{
Caracterización de pacientes con cáncer colorrectal esporádico basado en la nueva subclasificación molecular de consenso
}

\author{
ANA MARÍA WIELANDT ${ }^{1, a}$, CYNTHIA VILLARROEL ${ }^{1, a}$, \\ CLAUDIA HURTADO $^{1, \mathrm{~b}}$, DANIELA SIMIAN ${ }^{2, \mathrm{c}}$, DIEGO ZAMORANO ${ }^{4}$, \\ MARIPAZ MARTÍNEZ ${ }^{2, \mathrm{c}}$, MAGDALENA CASTRO ${ }^{2, \mathrm{~d}}$, MARÍA TERESA VIAL ${ }^{3}$, \\ UDO KRONBERG ${ }^{4}$, FRANCISCO LÓPEZ-KOSTNER ${ }^{4}$
}

\section{Characterization of patients with sporadic colorectal cancer following the new Consensus Molecular Subtypes (CMS)}

Background: Colorectal cancer (CRC) is an heterogeneous disease. Three carcinogenic pathways determine its molecular profile: microsatellite instability (MSI), chromosomal instability (CIN) and CpG island methylator phenotype (CIMP). Based on the new molecular classification, four consensus CRC molecular subtypes (CMS) are established, which are related to clinical, pathological and biological characteristics of the tumor. Aim: To classify Chilean patients with sporadic CRC according to the new consensus molecular subtypes of carcinogenic pathways. Material and Methods: Prospective analytical study of 53 patients with a mean age of 70 years (55\% males) with CRC, operated at a private clinic, without neoadjuvant treatment. From normal and tumor tissue DNA of each patient, CIN, MSI and CIMP were analyzed. Combining these variables, tumors were classified as CMS1/MSI-immune, CMS2/canonical, CMS3/metabolic and CMS4/mesenchymal. Results: CMS1 tumors (19\%) were located in the right colon, were in early stages, had MMR complex deficiencies and $67 \%$ had an activating mutation of the BRAF oncogene. CMS2 tumors (31\%) were located in the left colon, had moderate differentiation, absence of vascular invasion, lymphatic and mucin. CMS3 tumors (29\%) were also left-sided, with absence of vascular and lymphatic invasion, and $29 \%$ had an activating mutation of the KRAS oncogene. CMS4 tumors (21\%) showed advanced stages and presence of metastases. Conclusions: This new molecular classification contributes to understanding the heterogeneity of tumors. It is possible to differentiate molecular subgroups of a single pathological diagnosis of adenocarcinoma, opening the door to personalized medicine.

(Rev Med Chile 2017; 145: 419-430)

Key words: Carcinogenesis; Chromosomal Instability; Colorectal Neoplasms; Cp G Island; Microsatellite Instability.

$\mathrm{E}$ 1 cáncer colorrectal (CCR) representa un importante problema de salud a nivel mundial, ocupando el tercer lugar entre los cánceres más frecuentes y el cuarto en mortalidad por cáncer ${ }^{1,2}$. En Chile, se ha duplicado

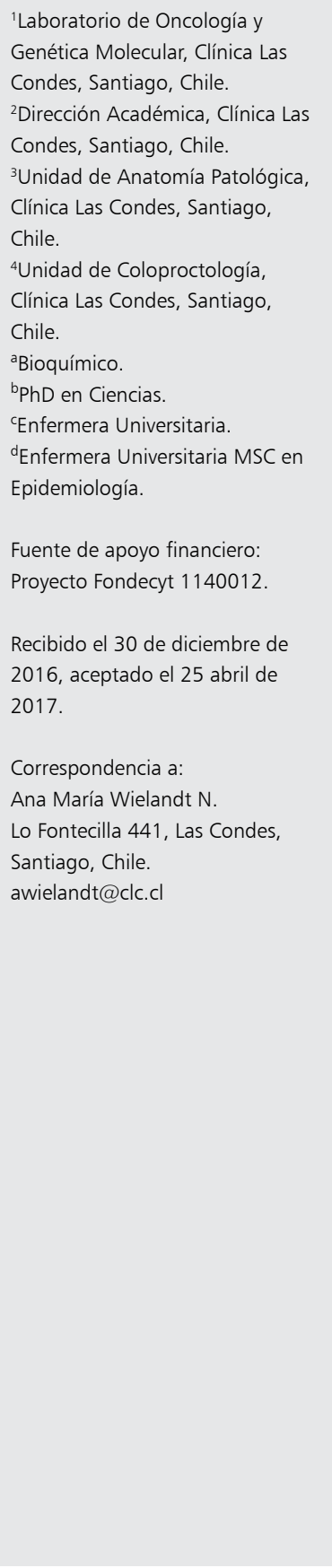

'Laboratorio de Oncología y

Genética Molecular, Clínica Las

Condes, Santiago, Chile.

Condes, Santiago, Chile.

3Unidad de Anatomía Patológica,

Clínica Las Condes, Santiago,

Chile.

¿Unidad de Coloproctología,

Clínica Las Condes, Santiago,

'Enfermera Universitaria.

dEnfermera Universitaria MSC en

Fuente de apoyo financiero:

Proyecto Fondecyt 1140012.

Recibido el 30 de diciembre de 2016, aceptado el 25 abril de

Lo Fontecilla 441, Las Condes,

Santiago, Chile.

awielandt@clc.cl su mortalidad en los últimos años a pesar de los programas de prevención primaria, ocupando el cuarto lugar de muerte por cáncer en hombres y el quinto en mujeres ${ }^{3}$.

En los últimos años, el modelo para CCR 
formulado por Vogelstein ${ }^{4}$ para la secuencia adenoma-carcinoma basado en la inestabilidad de diversos genes ha evolucionado ${ }^{5}$. Hoy en día, se ha determinado que el CCR es una enfermedad heterogénea y surge por la acumulación secuencial de procesos genéticos y epigenéticos ${ }^{6,7}$. Se ha descrito que el CCR esporádico se desarrolla principalmente por 3 eventos moleculares carcinogénicos: la inestabilidad cromosómica (CIN), la inestabilidad microsatelital (MSI) y el fenotipo metilador (CIMP) $)^{8}$.

El mecanismo CIN ocurre en $65 \%-70 \%$ de los casos de CCR esporádico. Permite identificar ganancias o pérdidas cromosomales y rearreglos estructurales que promueven la carcinogénesis a través de la pérdida de genes supresores de tumores o aumento en el número de copias de protooncogenes. Una de las formas de evaluarla es mediante la pérdida de heterocigosidad ( $\mathrm{LOH})$. La vía CIN ha sido propuesta como marcador predictivo en pacientes con etapas II y III ${ }^{9,10}$.

La MSI es causada por la incapacidad de la célula cancerosa en corregir deleciones o inserciones en regiones repetitivas del $\mathrm{ADN}$ por el complejo de reparación "mismatch repair" (MMR). Los defectos del complejo MMR (dMMR) se deben a mutaciones o deleciones en los genes que lo conforman o la hipermetilación en la región promotora del gen MLH1. Se observa en aproximadamente $15 \%$ $20 \%$ de los casos de CCR esporádicos y se asocia a buen pronóstico ${ }^{11,12}$.

La vía CIMP se caracteriza por una amplia hipermetilación de los islotes CpG en los promotores de genes supresores de tumores, provocando la inactivación de ellos. Se presenta en 15\%-20\% de CCR esporádicos y se le asocia a mal pronóstico $^{13,14}$.

Debido a la heterogeneidad y complejidad de los tumores, diversos estudios han propuesto subgrupos moleculares de CCR basados en las 3 vías antes descritas ${ }^{15-19}$. Los esfuerzos en determinar el perfil molecular de CCR tienen como fin comprender mejor los mecanismos que determinan el comportamiento clínico de los diferentes tumores y, de esta manera, identificar biomarcadores que proporcionen información pronóstica precoz. En base a ello, se podrá confeccionar un tratamiento personalizado a los pacientes, con el fin de mejorar su sobrevida. La subtipificación de CCR más reciente se realizó por un grupo de especialistas de varios países, que constituyeron The Colorectal Cancer Subtyping Consortium (CRCSC), en el año 2015, donde se logró identificar 4 subtipos moleculares por consenso definidos en CMS 1 a 4, según las características clínico-patológicas, vías moleculares involucradas y estado mutacional de los genes KRAS, BRAF y PI3KCA, con el fin de favorecer el tratamiento clínico ${ }^{19}$. Cabe notar que en ese estudio, $21 \%$ de los tumores no lograron ser categorizados en estos cuatro subtipos.

Dada la falta de subclasificación de nuestra población, se propuso realizar un estudio exploratorio del perfil molecular de las vías carcinogénicas CIN, MSI y CIMP en pacientes con CCR esporádico sometidos a cirugía en Clínica Las Condes para determinar la prevalencia de estos 4 subtipos.

\section{Materiales y Métodos}

\section{Diseño del estudio}

Se realizó un estudio analítico exploratorio prospectivo de 53 pacientes sometidos a cirugía por neoplasias colorrectales entre los años 2010 y 2016 en Clínica Las Condes (Figura 1). Los criterios de inclusión correspondieron a pacientes tratados por neoplasias colorrectales sin terapia neoadyuvante; como criterios de exclusión se consideraron pacientes con enfermedad inflamatoria, síndromes hereditarios de CCR o tratados previamente con radio/quimioterapia. Cada paciente firmó un consentimiento informado previamente aprobado por el Comité de Bioética de Clínica Las Condes.

A partir de los datos disponibles de la ficha clínica de los pacientes, se elaboró una base de datos con características demográficas y clínico-patológicas (Tabla 1).

\section{Extracción de ADN}

La extracción de ADN genómico normal se realizó a partir de una muestra de sangre venosa periférica por método Lahiri ${ }^{20}$.

El ADN tumoral se extrajo mediante el kit de extracción QIAmp DNA FFPE Tissue de QIAGEN (Hilden, Alemania), a partir de secciones de tejido tumoral fijado en formalina y embebido en parafina (FFPE); se seleccionaron áreas de la muestra con células tumorales $\geq 80 \%$. La integridad del ADN se verificó mediante reacción en cadena polimerasa (PCR) multiplex. 


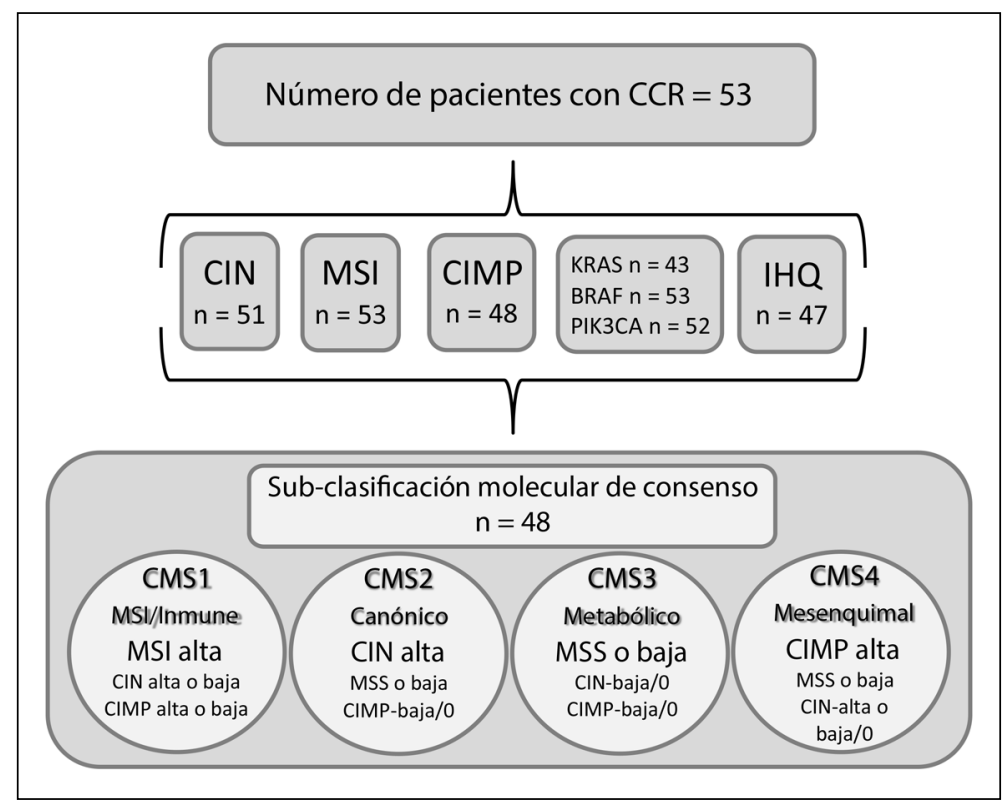

Figura 1. Flujograma de pacientes con cáncer colorrectal disponibles para el análisis de las distintas vías carcinogénicas. CCR: cáncer colorrectal; CIN: inestabilidad cromosomal; MSI: inestabilidad microsatelital; CIMP: fenotipo metilador de islotes CpG; MSS, MSI estable; IHQ: inmunohistoquímica; CMS: subtipo molecular consenso.

\section{Análisis del estado CIN}

Se realizó un análisis de deleción de 10 STRs (short tandem repeat) aledaños a los genes APC (D5S134-D5S346-D5S656-D5S82), DCC (D18S46-D18S64-D18S69) y P53 (D17S1176D17S1881-D17S250) mediante PCR $^{21}$. Cada marcador microsatelital fue amplificado por PCR a partir de ADN tumoral y normal del mismo paciente. Se consideró $\mathrm{LOH}$ positivo si la altura relativa del peak del ADN tumoral disminuye 30\% con respecto al ADN normal. El locus se consideró positivo si al menos uno de los marcadores informativos mostró LOH. Se considera CIN-alta si presenta sobre 3 STRs con pérdida alélica ${ }^{22}$.

\section{Análisis del estado de MSI}

Se determinó mediante el panel de siete marcadores microsatelitales recomendados por el National Cancer Institute (NCI) (Bat-25/Bat-26/ Bat-40/D2S123/D3S1029/D5S346/ D17S250). Cada marcador microsatelital fue amplificado mediante PCR a partir de ADN tumoral y normal del mismo paciente ${ }^{23}$. Los tumores se clasificaron en tres categorías: MSI-alta ( $\geq 3$ marcadores inestables), MSI-baja (1-2 marcadores inestables) y MSS (sin inestabilidad).

\section{CIMP}

Se determinó utilizando MethyLight ${ }^{24}$ para un panel de 6 marcadores (CACNA1G/IGF2/NEU-
ROG1/RUNX3/SOCS1/MLH1) ${ }^{25,26}$. Las muestras de ADN tumoral fueron modificadas mediante el kit EZ DNA Methylation-Gold (Zymo Research, Irvine, AC, USA). Se utilizó como gen de referencia ALU-C4. Como control positivo se usó ADN metilado con enzima SssI metiltransferasa (Promega, Madison, WI, USA). Se consideró un gen metilado si presentaba sobre $80 \%$ de metilación respecto al $\mathrm{ADN}$ control. Los tumores se clasifican en 3 categorías: CIMP-alta ( $\geq 3$ genes metilados), CIMP-baja ( 1 o 2 genes metilados) y CIMP-0 (sin genes metilados).

\section{Análisis de mutaciones somáticas en KRAS, BRAF y PIK3CA}

El ADN tumoral se analizó mediante PCR para las mutaciones más frecuentes en los genes KRAS (codón 12 y 13), BRAF (codón 600) y PIK3CA (exones 9 y 20$)^{27}$. Luego fueron analizados directamente por secuenciación Sanger.

\section{Análisis de inmunohistoquímica (IHQ)}

La evaluación de la expresión en el tumor de las proteínas del complejo MMR: MLH1, MSH2, MSH6 y PMS2 fue realizada por IHQ en tejidos $\mathrm{FFPE}^{23}$. Para la interpretación de los resultados, se consideró una variable dicotómica positiva y negativa para la presencia o ausencia de las proteínas, respectivamente. 
Tabla 1. Caracterización clínico-patológica de los pacientes con CCR analizados

\begin{tabular}{|c|c|c|c|c|c|}
\hline & n (\%) & $\begin{array}{c}\text { Hombres } \\
29(55)\end{array}$ & $\begin{array}{l}\text { Mujeres } \\
24(45)\end{array}$ & $\begin{array}{l}\text { Total (n) } \\
53(100)\end{array}$ & Valor $p^{a}$ \\
\hline Edad (años) & Mediana (mín-máx) & $69(50-89)$ & $70(41-97)$ & $70(41-97)$ & $0,7005^{b}$ \\
\hline Localización & $\begin{array}{l}\text { Derecho } \\
\text { Izquierdo-recto }\end{array}$ & $\begin{array}{r}6(21) \\
23(79)\end{array}$ & $\begin{array}{l}14(58) \\
10(42)\end{array}$ & $\begin{array}{l}20(38) \\
33(62)\end{array}$ & $0,0055^{*}$ \\
\hline Invasión vascular & $\begin{array}{l}\text { Positiva } \\
\text { Negativa }\end{array}$ & $\begin{array}{l}11(38) \\
18(62)\end{array}$ & $\begin{array}{r}5(21) \\
19(79)\end{array}$ & $\begin{array}{l}16(30) \\
37(70)\end{array}$ & 0,147 \\
\hline Invasión linfática & $\begin{array}{l}\text { Positiva } \\
\text { Negativa } \\
\mathrm{NI}\end{array}$ & $\begin{array}{r}9(31) \\
20(69) \\
0 \quad(0)\end{array}$ & $\begin{array}{r}8(33) \\
15(63) \\
1 \quad(4)\end{array}$ & $\begin{array}{r}17(32) \\
35(66) \\
1 \quad(2)\end{array}$ & 1 \\
\hline Invasión de pared & $\begin{array}{l}\text { pT1-T2 } \\
\text { pT3-T4 }\end{array}$ & $\begin{array}{r}4(14) \\
25(86)\end{array}$ & $\begin{array}{r}4(17) \\
20(83)\end{array}$ & $\begin{array}{r}8(15) \\
45(85)\end{array}$ & 0,1108 \\
\hline Estadio & $\begin{array}{l}\text { I-II } \\
\text { III-IV }\end{array}$ & $\begin{array}{l}13(45) \\
16(55)\end{array}$ & $\begin{array}{l}11(46) \\
13(54)\end{array}$ & $\begin{array}{l}24(45) \\
29(55)\end{array}$ & 1 \\
\hline Diferenciación & $\begin{array}{l}\text { Bien-moderada } \\
\text { Pobre }\end{array}$ & $\begin{array}{r}25(86) \\
4(14)\end{array}$ & $\begin{array}{r}20(83) \\
4(17)\end{array}$ & $\begin{array}{r}45(85) \\
8(15)\end{array}$ & 1 \\
\hline IMC (mín-máx) & $\begin{array}{l}\geq 25(25,09-36,23) \\
<25(18,73-24,65)\end{array}$ & $\begin{array}{r}20(69) \\
9(31)\end{array}$ & $\begin{array}{l}11(46) \\
13(54)\end{array}$ & $\begin{array}{l}31(58) \\
22(42)\end{array}$ & $0,0644^{b}$ \\
\hline Mucina & $\begin{array}{l}\text { Positivo } \\
\text { Negativo } \\
\mathrm{NI}\end{array}$ & $\begin{array}{r}7(24) \\
22(76) \\
0 \quad(0)\end{array}$ & $\begin{array}{r}8(33) \\
13(54) \\
3(13)\end{array}$ & $\begin{array}{c}15(28) \\
35(66) \\
3 \quad(6)\end{array}$ & 0,226 \\
\hline Tumor budding & $\begin{array}{l}\text { Bajo } \\
\text { Alto } \\
\mathrm{NI}\end{array}$ & $\begin{array}{r}17(59) \\
10(34) \\
2(7)\end{array}$ & $\begin{array}{r}17(71) \\
6(25) \\
1(4)\end{array}$ & $\begin{array}{r}34(64) \\
16(30) \\
3 \quad(6)\end{array}$ & 1 \\
\hline KRAS & $\begin{array}{l}\text { WT } \\
\text { MUT } \\
\text { ND }\end{array}$ & $\begin{array}{r}19(66) \\
6(21) \\
4(14)\end{array}$ & $\begin{array}{r}13(54) \\
5(21) \\
6(25)\end{array}$ & $\begin{array}{l}32(60) \\
11(21) \\
10(19)\end{array}$ & 1 \\
\hline BRAF & $\begin{array}{l}\text { WT } \\
\text { MUT }\end{array}$ & $\begin{array}{r}24(83) \\
5(17)\end{array}$ & $\begin{array}{r}21(88) \\
3(12)\end{array}$ & $\begin{array}{r}45(85) \\
8(15)\end{array}$ & 1 \\
\hline PIK3CA & $\begin{array}{l}\text { WT } \\
\text { MUT } \\
\text { ND }\end{array}$ & $\begin{array}{r}22(76) \\
6(21) \\
1 \quad(3)\end{array}$ & $\begin{array}{r}16(67) \\
8(33) \\
0 \quad(0)\end{array}$ & $\begin{array}{c}38(72) \\
14(26) \\
1 \quad(2)\end{array}$ & 0,2572 \\
\hline
\end{tabular}

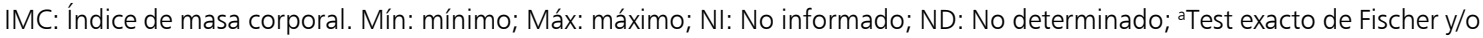
$\chi^{2}$ si no hay otra especificación; ${ }^{\mathrm{b}}$ Test Mann-Whitney; ${ }^{*} p<0,05$.

\section{Clasificación molecular}

El grupo CMS1 o inmune consideró pacientes que presentan MSI-alta independiente del estado de CIMP o CIN. En el grupo CMS2 o canónico se consideraron los tumores con CIN-alta, MSS/ baja y CIMP-baja/0. El Grupo CMS3 o metabólico consideró aquellos tumores con las vías MSI, CIMP y CIN baja o estables. En el grupo CMS4 o mesenquimal se categorizaron los tumores que presentaron CIMP-alta y MSS/baja ${ }^{19}$.

\section{Análisis estadístico}

Las variables categóricas (dicotómicas y policotómicas) se describieron con frecuencia absoluta y porcentual, las continuas, con mediana e intervalo mínimo-máximo, porque no distribuyeron normal. Para comparar las categóricas, se usó Test exacto de Fischer y/o $\chi^{2}$ dependiendo del menor o mayor a 30. Para contínuas test de Mann Whitney (2 grupos) o Kruskal Wallis (más de 2 grupos). Se utilizó el software estadístico STATA12. 


\section{Resultados}

\section{Descripción clínico-patológica}

Se analizaron un total de 53 pacientes, $55 \%$ hombres, con una mediana de edad de 70 años. Sesenta y dos por ciento de los pacientes tenía localizado el tumor en lado izquierdo. Respecto de las mutaciones somáticas, $21 \%(11 / 53)$ de los pacientes presentaron mutaciones activantes en el oncogen KRAS, 15\% (8/53) en BRAF y 26\% (14/53) en PI3KCA (Tabla 1).

\section{Análisis MSI}

Los tumores fueron categorizados en MSI-baja/MSS y MSI-alta con una prevalencia de $83 \%$ (44/53) y $17 \%$ (9/53), respectivamente (Tabla 2$)$. Los tumores con MSI-alta tuvieron diferencias significativas respecto a edad, ubicación, preferentemente en el lado derecho del colon, presencia de patrón de invasión linfática y pobre diferenciación comparado con tumores MSI-baja/MSS con una alta prevalencia de BRAF $^{\text {mut }}$ (Tabla 2).

Tabla 2. Análisis de la inestabilidad microsatelital (MSI) según las características clínico-patológicas y moleculares

\begin{tabular}{|c|c|c|c|c|c|}
\hline & n (\%) & $\begin{array}{l}\text { MSI-alta } \\
9 \text { (17) }\end{array}$ & $\begin{array}{c}\text { MSI-baja/MSS } \\
44(83)\end{array}$ & $\begin{array}{c}\text { Total } \\
53(100)\end{array}$ & Valor $\mathbf{p}^{\mathbf{a}}$ \\
\hline Género & $\begin{array}{l}\text { Femenino } \\
\text { Masculino }\end{array}$ & $\begin{array}{l}5(21) \\
4(14)\end{array}$ & $\begin{array}{ll}19 & (79) \\
25 & (86)\end{array}$ & $\begin{array}{l}24(100) \\
29(100)\end{array}$ & 0,3756 \\
\hline Edad (años) & $\begin{array}{l}<60 \\
\geq 60\end{array}$ & $\begin{array}{lr}0 & (0) \\
9 & (21)\end{array}$ & $\begin{array}{ll}10 & (23) \\
34 & (79)\end{array}$ & $\begin{array}{l}10(100) \\
43(100)\end{array}$ & $0,0426^{b *}$ \\
\hline Localización & $\begin{array}{l}\text { Derecho } \\
\text { Izquierdo-recto }\end{array}$ & $\begin{array}{l}8(40) \\
1 \quad(3)\end{array}$ & $\begin{array}{ll}12 & (60) \\
32 & (97)\end{array}$ & $\begin{array}{l}20(100) \\
33(100)\end{array}$ & $0,001^{*}$ \\
\hline Invasión vascular & $\begin{array}{l}\text { Positiva } \\
\text { Negativa }\end{array}$ & $\begin{array}{l}2(12) \\
7(19)\end{array}$ & $\begin{array}{ll}14 & (88) \\
30 & (81)\end{array}$ & $\begin{array}{l}16(100) \\
37(100)\end{array}$ & 0,4462 \\
\hline Invasión linfática & $\begin{array}{l}\text { Positiva } \\
\text { Negativa } \\
\text { NI }\end{array}$ & $\begin{array}{lr}6 & (35) \\
3 & (9) \\
0 & (0)\end{array}$ & $\begin{array}{rr}11 & (65) \\
32 & (91) \\
1 & (100)\end{array}$ & $\begin{array}{r}17(100) \\
35(100) \\
1(100)\end{array}$ & $0,0254^{*}$ \\
\hline Invasión de pared & $\begin{array}{l}\text { pT1-T2 } \\
\text { pT3-T4 }\end{array}$ & $\begin{array}{l}1(12) \\
8(18)\end{array}$ & $\begin{array}{rr}7 & (88) \\
37 & (82)\end{array}$ & $\begin{array}{r}8(100) \\
45(100)\end{array}$ & 0,651 \\
\hline Estadio & $\begin{array}{l}\text { I-II } \\
\text { III-IV }\end{array}$ & $\begin{array}{l}5(21) \\
4(14)\end{array}$ & $\begin{array}{ll}19 & (79) \\
25 & (86)\end{array}$ & $\begin{array}{l}24(100) \\
29(100)\end{array}$ & 0,3756 \\
\hline Diferenciación & $\begin{array}{l}\text { Bien-moderada } \\
\text { Pobre }\end{array}$ & $\begin{array}{l}5(11) \\
4(50)\end{array}$ & $\begin{array}{rr}40 & (89) \\
4 & (50)\end{array}$ & $\begin{array}{r}45(100) \\
8(100)\end{array}$ & $0,0213^{*}$ \\
\hline IMC (mín-máx) & $\begin{array}{l}\geq 25(25,09-36,23) \\
<25(18,73-24,65)\end{array}$ & $\begin{array}{l}5(16) \\
4(18)\end{array}$ & $\begin{array}{ll}26 & (84) \\
18 & (82)\end{array}$ & $\begin{array}{l}31(100) \\
22(100)\end{array}$ & $0,5458^{b}$ \\
\hline Mucina & $\begin{array}{l}\text { Positiva } \\
\text { Negativa } \\
\text { NI }\end{array}$ & $\begin{array}{l}4(27) \\
4(11) \\
1(33)\end{array}$ & $\begin{aligned} 11 & (73) \\
31 & (88) \\
2 & (67)\end{aligned}$ & $\begin{array}{r}15(100) \\
35(100) \\
3(100)\end{array}$ & 0,1757 \\
\hline Tumor budding & $\begin{array}{l}\text { Bajo } \\
\text { Alto } \\
\mathrm{NI}\end{array}$ & $\begin{array}{l}5(15) \\
3(19) \\
1(33)\end{array}$ & $\begin{aligned} 29 & (85) \\
13 & (81) \\
2 & (67)\end{aligned}$ & $\begin{array}{r}34(100) \\
16(100) \\
3(100)\end{array}$ & 0,0578 \\
\hline KRAS & $\begin{array}{l}\text { WT } \\
\text { MUT } \\
\text { ND }\end{array}$ & $\begin{array}{l}5(16) \\
1 \quad(9) \\
3(30)\end{array}$ & $\begin{aligned} 27 & (84) \\
10 & (91) \\
7 & (70)\end{aligned}$ & $\begin{array}{l}32(100) \\
11(100) \\
10(100)\end{array}$ & 0,512 \\
\hline BRAF & $\begin{array}{l}\text { WT } \\
\text { MUT }\end{array}$ & $\begin{array}{l}3 \quad(7) \\
6(75)\end{array}$ & $\begin{array}{rr}42 & (93) \\
2 & (25)\end{array}$ & $\begin{array}{r}45(100) \\
8(100)\end{array}$ & $<0,001^{*}$ \\
\hline PIK3CA & $\begin{array}{l}\text { WT } \\
\text { MUT } \\
\text { ND }\end{array}$ & $\begin{array}{l}7(18) \\
2(14) \\
0 \quad(0)\end{array}$ & $\begin{array}{rr}31 & (82) \\
12 & (86) \\
1 & (100)\end{array}$ & $\begin{array}{r}38(100) \\
14(100) \\
1(100)\end{array}$ & 0,5861 \\
\hline
\end{tabular}

MSI: inestabilidad microsatelital; MSS, MSI estable; IMC: Índice de masa corporal. Mín: mínimo; Máx: máximo; NI: No informado; ND: No determinado; ${ }^{a}$ Test exacto de Fischer y/o Test $\chi^{2}$ si no hay otra especificación; ${ }^{b} T e s t$ Mann-Whitney; ${ }^{*} p<0,05$. 


\section{Análisis CIMP}

Sólo en 48 pacientes se logró tener ADN suficiente para determinar el estado CIMP. El 31\% (15/48) de los tumores presentó CIMP-alta y 69\% CIMP-baja/0. Los tumores CIMP-alta se ubicaron frecuentemente al lado derecho del colon y presentaron pobre grado de diferenciación comparado con tumores CIMP-baja/0 y alta presencia de BRAF $^{\text {mut }}$ (Tabla 3).

\section{Análisis CIN}

Se logró analizar el estado CIN en 51 pacientes, $10 \%(5 / 51)$ de los casos analizados fueron no informativos por presentar inestabilidad microsatelital.

Tabla 3. Análisis del fenotipo metilador (CIMP) según las características clínico-patológicas y moleculares

\begin{tabular}{|c|c|c|c|c|c|}
\hline & n (\%) & $\begin{array}{l}\text { CIMP-alta } \\
15(31)\end{array}$ & $\begin{array}{l}\text { CIMP-baja/0 } \\
33 \text { (69) }\end{array}$ & $\begin{array}{c}\text { Total } \\
48(100)\end{array}$ & Valor $\mathbf{p}^{\mathbf{a}}$ \\
\hline Género & $\begin{array}{l}\text { Femenino } \\
\text { Masculino }\end{array}$ & $\begin{array}{l}6(27) \\
9(35)\end{array}$ & $\begin{array}{ll}16 & (73) \\
17 & (65)\end{array}$ & $\begin{array}{l}22(100) \\
26(100)\end{array}$ & 0,4089 \\
\hline Edad (años) & $\begin{array}{l}<60 \\
\geq 60\end{array}$ & $\begin{array}{r}1(10) \\
14(37)\end{array}$ & $\begin{array}{rr}9 & (90) \\
24 & (63)\end{array}$ & $\begin{array}{l}10(100) \\
38(100)\end{array}$ & $0,1737^{b}$ \\
\hline Localización & $\begin{array}{l}\text { Derecho } \\
\text { Izquierdo-recto }\end{array}$ & $\begin{array}{l}9(53) \\
6(19)\end{array}$ & $\begin{array}{rr}8 & (47) \\
25 & (81)\end{array}$ & $\begin{array}{l}17(100) \\
31(100)\end{array}$ & $0,0198^{*}$ \\
\hline Invasión vascular & $\begin{array}{l}\text { Positiva } \\
\text { Negativa }\end{array}$ & $\begin{array}{l}6(43) \\
9(26)\end{array}$ & $\begin{array}{rr}8 & (57) \\
25 & (74)\end{array}$ & $\begin{array}{l}14(100) \\
34(100)\end{array}$ & 0,2186 \\
\hline Invasión linfática & $\begin{array}{l}\text { Positiva } \\
\text { Negativa } \\
\mathrm{NI}\end{array}$ & $\begin{array}{l}6(40) \\
9(28) \\
0 \quad(0)\end{array}$ & $\begin{array}{lr}9 & (60) \\
2 & (72) \\
1 & (100)\end{array}$ & $\begin{array}{r}15(100) \\
32(100) \\
1(100)\end{array}$ & 0,3126 \\
\hline Invasión de pared & $\begin{array}{l}\text { pT1-T2 } \\
\text { pT3-T4 }\end{array}$ & $\begin{array}{r}3(38) \\
12(30)\end{array}$ & $\begin{aligned} 5 & (62) \\
28 & (70)\end{aligned}$ & $\begin{array}{r}8(100) \\
40(100)\end{array}$ & 0,3941 \\
\hline Estadio & $\begin{array}{l}\text { I-II } \\
\text { III-IV }\end{array}$ & $\begin{array}{r}5(23) \\
10(38)\end{array}$ & $\begin{array}{ll}17 & (77) \\
16 & (62)\end{array}$ & $\begin{array}{l}22(100) \\
26(100)\end{array}$ & 0,1956 \\
\hline Diferenciación & $\begin{array}{l}\text { Bien-moderada } \\
\text { Pobre }\end{array}$ & $\begin{array}{l}9(22) \\
6(86)\end{array}$ & $\begin{array}{rr}32 & (78) \\
1 & (14)\end{array}$ & $\begin{array}{r}41(100) \\
7(100)\end{array}$ & $0,0026^{*}$ \\
\hline IMC (mín-máx) & $\begin{array}{l}\geq 25(25,09-36,23) \\
<25(18,73-24,65)\end{array}$ & $\begin{array}{l}9(35) \\
6(27)\end{array}$ & $\begin{array}{ll}17 & (65) \\
16 & (73)\end{array}$ & $\begin{array}{l}26(100) \\
22(100)\end{array}$ & $0,3732^{b}$ \\
\hline Mucina & $\begin{array}{l}\text { Positiva } \\
\text { Negativa } \\
\text { NI }\end{array}$ & $\begin{array}{r}3(21) \\
11(35) \\
1(33)\end{array}$ & $\begin{array}{rr}11 & (79) \\
20 & (65) \\
2 & (67)\end{array}$ & $\begin{array}{r}14(100) \\
31(100) \\
3(100)\end{array}$ & 0,2805 \\
\hline Tumor budding & $\begin{array}{l}\text { Bajo } \\
\text { Alto } \\
\mathrm{NI}\end{array}$ & $\begin{array}{r}10(30) \\
4(33) \\
1(33)\end{array}$ & $\begin{aligned} 23 & (70) \\
8 & (67) \\
2 & (67)\end{aligned}$ & $\begin{array}{r}33(100) \\
12(100) \\
3(100)\end{array}$ & 0,5584 \\
\hline Metástasis & $\begin{array}{l}\text { Negativa } \\
\text { Positiva } \\
\text { Otro primario } \\
\mathrm{NI}\end{array}$ & $\begin{array}{l}8(24) \\
4(67) \\
2(50) \\
1(25)\end{array}$ & $\begin{aligned} 26 & (76) \\
2 & (33) \\
2 & (50) \\
3 & (75)\end{aligned}$ & $\begin{array}{r}34(100) \\
6(100) \\
4(100) \\
4(100)\end{array}$ & 0,0803 \\
\hline KRAS & $\begin{array}{l}\text { WT } \\
\text { MUT } \\
\text { ND }\end{array}$ & $\begin{array}{l}9(31) \\
2(20) \\
4(44)\end{array}$ & $\begin{aligned} 20 & (69) \\
8 & (80) \\
5 & (56)\end{aligned}$ & $\begin{array}{r}29(100) \\
10(100) \\
9(100)\end{array}$ & 0,409 \\
\hline BRAF & $\begin{array}{l}\text { WT } \\
\text { MUT }\end{array}$ & $\begin{array}{r}10(24) \\
5(71)\end{array}$ & $\begin{aligned} 31 & (76) \\
2 & (29)\end{aligned}$ & $\begin{array}{r}41(100) \\
7(100)\end{array}$ & $0,0239 *$ \\
\hline PIK3CA & $\begin{array}{l}\text { WT } \\
\text { MUT } \\
\text { ND }\end{array}$ & $\begin{array}{r}12(35) \\
3(23) \\
0 \quad(0)\end{array}$ & $\begin{array}{rr}22 & (65) \\
10 & (77) \\
1 & (100)\end{array}$ & $\begin{array}{r}34(100) \\
13(100) \\
1(100)\end{array}$ & 0,3917 \\
\hline
\end{tabular}

CIMP, Fenotipo metilador de los islotes CpG; IMC: Índice de masa corporal; Mín: mínimo; Máx: máximo; NI: No informado; ND: No determinado; ${ }^{a}$ Test exacto de Fischer y/o $\chi^{2}$ si no hay otra especificación; ${ }^{b} T e s t$ Mann-Whitney; * $p<0,05$. 
De las 46 muestras restantes, 50\% fueron CIN-baja/0 y 50\% CIN-alta. Los tumores con CIN-alta, al momento del diagnóstico, se encontraban en estadíos más avanzados, comparado con tumores CIN-baja/0 y eran no mucinosos (Tabla 4 ).

\section{Clasificación molecular}

Se identificaron los 4 grupos establecidos por el CRCSC. El 9\% de los pacientes quedó sin clasificar.
$\mathrm{Al}$ analizar las características clínico-patológicas, se observó que en los tumores CMS1 (19\%) todos los pacientes eran $\geq 60$ años (Tabla 5); se ubicaban en el lado derecho del colon, tenían MLH1 hipermetilado, mucina, frecuente invasión linfática, estadios tempranos y alta prevalencia de oncogen BRAF $^{\text {mut }}, 7 / 9$ pacientes presentaban mutaciones en alguno de los oncogenes. En 2 de los casos existía concomitancia de $\mathrm{BRAF}^{\mathrm{mut}}$ y PI3KCA ${ }^{\mathrm{mut}}$.

Tabla 4. Análisis de la inestabilidad cromosomal (CIN) según las características clínico-patológicas y moleculares

\begin{tabular}{|c|c|c|c|c|c|}
\hline & n (\%) & $\begin{array}{r}\text { CIN-alta } \\
23(50)\end{array}$ & $\begin{array}{r}\text { CIN-baja } \\
23(50)\end{array}$ & $\begin{array}{c}\text { Total } \\
46(100)\end{array}$ & Valor $\boldsymbol{p}^{\mathbf{a}}$ \\
\hline Género & $\begin{array}{l}\text { Femenino } \\
\text { Masculino }\end{array}$ & $\begin{array}{ll}10 & (50) \\
13 & (50)\end{array}$ & $\begin{array}{ll}10 & (50) \\
13 & (50)\end{array}$ & $\begin{array}{l}20(100) \\
26(100)\end{array}$ & 1 \\
\hline Edad (años) & $\begin{array}{l}<60 \\
\geq 60\end{array}$ & $\begin{array}{rr}7 & (70) \\
16 & (44)\end{array}$ & $\begin{array}{rr}3 & (30) \\
20 & (56)\end{array}$ & $\begin{array}{l}10(100) \\
36(100)\end{array}$ & $0,1084^{b}$ \\
\hline Localización & $\begin{array}{l}\text { Derecho } \\
\text { Izquierdo-recto }\end{array}$ & $\begin{array}{rr}5 & (36) \\
18 & (56)\end{array}$ & $\begin{array}{rr}9 & (64) \\
14 & (44)\end{array}$ & $\begin{array}{l}14(100) \\
32(100)\end{array}$ & 0,1684 \\
\hline Invasión vascular & $\begin{array}{l}\text { Positiva } \\
\text { Negativa }\end{array}$ & $\begin{array}{rr}7 & (50) \\
16 & (50)\end{array}$ & $\begin{array}{rr}7 & (50) \\
16 & (50)\end{array}$ & $\begin{array}{l}14(100) \\
32(100)\end{array}$ & 1 \\
\hline Invasión linfática & $\begin{array}{l}\text { Positiva } \\
\text { Negativa } \\
\mathrm{NI}\end{array}$ & $\begin{array}{rr}6 & (43) \\
16 & (52) \\
1 & (100)\end{array}$ & $\begin{array}{rr}8 & (57) \\
15 & (48) \\
0 & (0)\end{array}$ & $\begin{array}{r}14(100) \\
31(100) \\
1(100)\end{array}$ & 0,4129 \\
\hline Invasión de pared & $\begin{array}{l}\text { pT1-T2 } \\
\text { рT3-T4 }\end{array}$ & $\begin{array}{rr}6 & (86) \\
17 & (44)\end{array}$ & $\begin{array}{rr}1 & (14) \\
22 & (56)\end{array}$ & $\begin{array}{r}7(100) \\
39(100)\end{array}$ & 0,0835 \\
\hline Estadio & $\begin{array}{l}\text { I-II } \\
\text { III-IV }\end{array}$ & $\begin{array}{rr}8 & (36) \\
15 & (63)\end{array}$ & $\begin{array}{rr}14 & (64) \\
9 & (38)\end{array}$ & $\begin{array}{l}22(100) \\
24(100)\end{array}$ & 0,0696 \\
\hline Diferenciación & $\begin{array}{l}\text { Bien-moderada } \\
\text { Pobre }\end{array}$ & $\begin{array}{rr}22 & (52) \\
1 & (25)\end{array}$ & $\begin{array}{rr}20 & (48) \\
3 & (75)\end{array}$ & $\begin{array}{r}42(100) \\
4(100)\end{array}$ & 0,3209 \\
\hline IMC (mín-máx) & $\begin{array}{l}\geq 25(25,09-36,23) \\
<25(18,73-24,65)\end{array}$ & $\begin{array}{rr}14 & (50) \\
9 & (50)\end{array}$ & $\begin{array}{rr}14 & (50) \\
9 & (50)\end{array}$ & $\begin{array}{l}28(100) \\
18(100)\end{array}$ & $0,9562^{b}$ \\
\hline Mucina & $\begin{array}{l}\text { Positiva } \\
\text { Negativa } \\
\mathrm{NI}\end{array}$ & $\begin{aligned} 3 & (25) \\
19 & (59) \\
1 & (50)\end{aligned}$ & $\begin{aligned} 9 & (75) \\
13 & (41) \\
1 & (50)\end{aligned}$ & $\begin{array}{r}12(100) \\
32(100) \\
2(100)\end{array}$ & $0,0442^{*}$ \\
\hline Tumor budding & $\begin{array}{l}\text { Bajo } \\
\text { Alto } \\
\mathrm{NI}\end{array}$ & $\begin{array}{rr}15 & (48) \\
8 & (62) \\
0 & (0)\end{array}$ & $\begin{array}{r}16 \quad(52) \\
5 \quad(38) \\
2(100)\end{array}$ & $\begin{array}{r}31(100) \\
13(100) \\
2(100)\end{array}$ & 0,3217 \\
\hline KRAS & $\begin{array}{l}\text { WT } \\
\text { MUT } \\
\text { ND }\end{array}$ & $\begin{array}{rr}17 & (59) \\
3 & (27) \\
3 & (50)\end{array}$ & $\begin{array}{rr}12 & (41) \\
8 & (73) \\
3 & (50)\end{array}$ & $\begin{array}{r}29(100) \\
11(100) \\
6(100)\end{array}$ & 0,0776 \\
\hline BRAF & $\begin{array}{l}\text { WT } \\
\text { MUT }\end{array}$ & $\begin{aligned} 21 & (50) \\
2 & (50)\end{aligned}$ & $\begin{array}{rr}21 & (50) \\
2 & (50)\end{array}$ & $\begin{array}{r}42(100) \\
4(100)\end{array}$ & 1 \\
\hline PIK3CA & $\begin{array}{l}\text { WT } \\
\text { MUT } \\
\text { ND }\end{array}$ & $\begin{array}{r}16(48) \\
6 \quad(50) \\
1(100)\end{array}$ & $\begin{array}{rr}17 & (52) \\
6 & (50) \\
0 & (0)\end{array}$ & $\begin{array}{r}33(100) \\
12(100) \\
1(100)\end{array}$ & 0,5 \\
\hline
\end{tabular}

CIN: Inestabilidad cromosomal; IMC: Índice de masa corporal. Mín: mínimo; Máx: máximo; NI: No informado; ND: No determinado; ${ }^{a}$ Test exacto de Fischer y/o Test $\chi^{2}$ si no hay otra especificación; ${ }^{\mathrm{b} T e s t}$ Mann-Whitney; * $\mathrm{p}<0,05$. 
Tabla 5. Comparación de las variables clínico-patológicas y moleculares con los subgrupos consenso

\begin{tabular}{|c|c|c|c|c|c|c|c|}
\hline & n (\%) & $\begin{array}{l}\text { CMS1 } \\
9(19)\end{array}$ & $\begin{array}{c}\text { CMS2 } \\
15(31)\end{array}$ & $\begin{array}{c}\text { CMS3 } \\
14(29)\end{array}$ & $\begin{array}{c}\text { CMS4 } \\
10(21)\end{array}$ & $\begin{array}{c}\text { Total } \\
48(100)\end{array}$ & Valor $p^{a}$ \\
\hline Género & $\begin{array}{l}\text { Femenino } \\
\text { Masculino }\end{array}$ & $\begin{array}{l}5(22) \\
4(16)\end{array}$ & $\begin{array}{ll}8 & (35) \\
7 & (28)\end{array}$ & $\begin{array}{l}6(26) \\
8(32)\end{array}$ & $\begin{array}{l}4(17) \\
6(24)\end{array}$ & $\begin{array}{l}23(100) \\
25(100)\end{array}$ & 0,8539 \\
\hline Edad (años) & $\begin{array}{l}<60 \\
\geq 60\end{array}$ & $\begin{array}{lr}0 & (0) \\
9 & (23)\end{array}$ & $\begin{array}{ll}6 & (67) \\
9 & (23)\end{array}$ & $\begin{array}{r}2(22) \\
12(31)\end{array}$ & $\begin{array}{l}1(11) \\
9(23)\end{array}$ & $\begin{array}{r}9(100) \\
39(100)\end{array}$ & $0,0408^{c}$ \\
\hline Localización & $\begin{array}{l}\text { Derecho } \\
\text { Izquierdo-recto }\end{array}$ & $\begin{array}{l}8(42) \\
1 \quad(3)\end{array}$ & $\begin{array}{rr}2 & (11) \\
13 & (45)\end{array}$ & $\begin{array}{l}5(26) \\
9(31)\end{array}$ & $\begin{array}{l}4(21) \\
6(21)\end{array}$ & $\begin{array}{l}19(100) \\
29(100)\end{array}$ & $0,004^{*}$ \\
\hline Invasión vascular & $\begin{array}{l}\text { Negativa } \\
\text { Positiva }\end{array}$ & $\begin{array}{l}7(20) \\
2(15)\end{array}$ & $\begin{array}{rr}12 & (34) \\
3 & (23)\end{array}$ & $\begin{array}{r}10(29) \\
4(31)\end{array}$ & $\begin{array}{l}6(17) \\
4(31)\end{array}$ & $\begin{array}{l}35(100) \\
13(100)\end{array}$ & 0,718 \\
\hline Invasión linfática & $\begin{array}{l}\text { Negativa } \\
\text { Positiva } \\
\text { NI }\end{array}$ & $\begin{array}{l}3(10) \\
6(38) \\
0 \quad(0)\end{array}$ & $\begin{array}{rr}11 & (35) \\
3 & (19) \\
1 & (100)\end{array}$ & $\begin{array}{r}10(32) \\
4(25) \\
0 \quad(0)\end{array}$ & $\begin{array}{lr}7 & (23) \\
3 & (19) \\
0 & (0)\end{array}$ & $\begin{array}{r}31(100) \\
16(100) \\
1(100)\end{array}$ & 0,138 \\
\hline Estadio & $\begin{array}{l}\text { I } \\
\text { II } \\
\text { III } \\
\text { IV }\end{array}$ & $\begin{array}{l}1(17) \\
4(25) \\
2(11) \\
2(29)\end{array}$ & $\begin{array}{ll}3 & (50) \\
3 & (19) \\
6 & (32) \\
3 & (42)\end{array}$ & $\begin{array}{lr}0 & (0) \\
8 & (50) \\
6 & (32) \\
0 & (0)\end{array}$ & $\begin{array}{l}2(33) \\
1 \quad(6) \\
5(26) \\
2(29)\end{array}$ & $\begin{array}{r}6(100) \\
16(100) \\
19(40) \\
7(100)\end{array}$ & 0,217 \\
\hline Diferenciación & $\begin{array}{l}\text { Bien-moderada } \\
\text { Pobre }\end{array}$ & $\begin{array}{l}5(13) \\
4(50)\end{array}$ & $\begin{array}{rr}15 & (38) \\
0 & (0)\end{array}$ & $\begin{array}{r}13(32) \\
1(12)\end{array}$ & $\begin{array}{l}7(17) \\
3(38)\end{array}$ & $\begin{array}{r}40(100) \\
8(100)\end{array}$ & $0,017^{*}$ \\
\hline IMC (mín-máx) & $\begin{array}{l}\geq 25(25,09-36,23) \\
<25(18,73-24,65)\end{array}$ & $\begin{array}{l}5(18) \\
4(19)\end{array}$ & $\begin{array}{ll}8 & (30) \\
7 & (33)\end{array}$ & $\begin{array}{l}7(26) \\
7(33)\end{array}$ & $\begin{array}{l}7(26) \\
3(14)\end{array}$ & $\begin{array}{l}27(100) \\
21(100)\end{array}$ & 0,7579 \\
\hline Tumor budding & $\begin{array}{l}\text { Bajo } \\
\text { Alto } \\
\mathrm{NI}\end{array}$ & $\begin{array}{l}5(15) \\
3(23) \\
1(50)\end{array}$ & $\begin{array}{rr}10 & (30) \\
5 & (38) \\
0 & (0)\end{array}$ & $\begin{array}{r}11(33) \\
3(23) \\
0 \quad(0)\end{array}$ & $\begin{array}{l}7(22) \\
2(15) \\
1(50)\end{array}$ & $\begin{array}{r}33(100) \\
13(100) \\
2(100)\end{array}$ & 0,7985 \\
\hline Mucina & $\begin{array}{l}\text { Negativa } \\
\text { Positiva } \\
\mathrm{NI}\end{array}$ & $\begin{array}{l}4(13) \\
4(27) \\
1(50)\end{array}$ & $\begin{aligned} 11 & (35) \\
3 & (20) \\
1 & (50)\end{aligned}$ & $\begin{array}{l}7(22) \\
7(47) \\
0 \quad(0)\end{array}$ & $\begin{array}{lr}9 & (29) \\
1 & (6) \\
0 & (0)\end{array}$ & $\begin{array}{l}31(100) \\
15(31) \\
2(100)\end{array}$ & 0,121 \\
\hline Metástasis & $\begin{array}{l}\text { Negativa } \\
\text { Sincrónica } \\
\text { Otro primario } \\
\mathrm{NI}\end{array}$ & $\begin{array}{ll}6 & (19) \\
2 & (24) \\
1 & (25) \\
0 & (0)\end{array}$ & $\begin{array}{ll}9 & (28) \\
3 & (38) \\
1 & (25) \\
2 & (50)\end{array}$ & $\begin{array}{r}12(37) \\
0(0) \\
1(25) \\
1(25)\end{array}$ & $\begin{array}{l}5(16) \\
3(38) \\
1(25) \\
1(25)\end{array}$ & $\begin{array}{r}32(100) \\
8(100) \\
4(100) \\
4(100)\end{array}$ & 0,543 \\
\hline KRAS & $\begin{array}{l}\text { WT } \\
\text { MUT } \\
\text { ND }\end{array}$ & $\begin{array}{l}5(17) \\
1(10) \\
3(34)\end{array}$ & $\begin{aligned} 10 & (34) \\
3 & (30) \\
2 & (22)\end{aligned}$ & $\begin{array}{l}8(28) \\
4(40) \\
2(22)\end{array}$ & $\begin{array}{l}6(21) \\
2(20) \\
2(22)\end{array}$ & $\begin{array}{r}29(100) \\
10(100) \\
9(100)\end{array}$ & 0,8797 \\
\hline BRAF & $\begin{array}{l}\text { WT } \\
\text { MUT }\end{array}$ & $\begin{array}{l}3 \quad(8) \\
6(76)\end{array}$ & $\begin{array}{rr}14 & (35) \\
1 & (12)\end{array}$ & $\begin{array}{r}14(35) \\
0 \quad(0)\end{array}$ & $\begin{array}{l}9(22) \\
1(12)\end{array}$ & $\begin{array}{r}40(100) \\
8(100)\end{array}$ & $0,0001^{*}$ \\
\hline PIK3CA & $\begin{array}{l}\text { WT } \\
\text { MUT } \\
\text { ND }\end{array}$ & $\begin{array}{l}7(20) \\
2(17) \\
0 \quad(0)\end{array}$ & $\begin{array}{rr}10 & (29) \\
4 & (33) \\
1 & (100)\end{array}$ & $\begin{array}{r}10(29) \\
4(33) \\
0 \quad(0)\end{array}$ & $\begin{array}{l}8(22) \\
2(17) \\
0 \quad(0)\end{array}$ & $\begin{array}{r}35(100) \\
12(100) \\
1(100)\end{array}$ & 0,9506 \\
\hline
\end{tabular}

CMS: Subtipo Molecular Consenso; IMC: Índice de masa corporal; Mín: mínimo; Máx: máximo; NI: No informado; ND: No

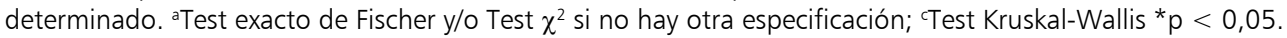

Tenían CIMP-alta a diferencia de CMS2 y CMS3 (Figura 2).

El grupo CMS2 presentó principalmente pacientes cuya edad diagnóstica fue $<60$ años. Representó el grupo más frecuente (31\%), se lo- calizaron al lado izquierdo del colon, con ausencia de invasión vascular, linfática y mucina y todos ellos con grado bien/moderado de diferenciación (Tabla 5, Figura 2).

Los tumores CMS3 (29\%) se presentaron 


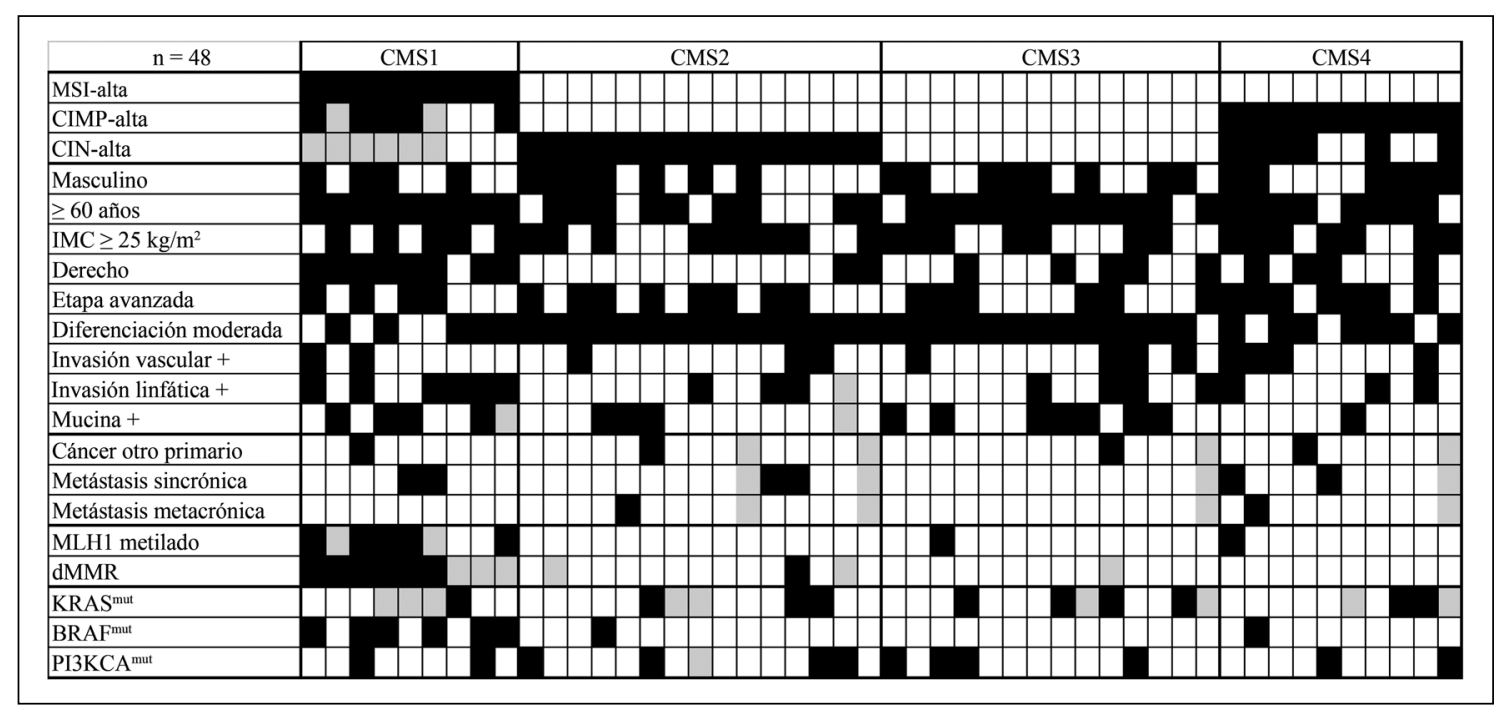

Figura 2. Análisis comparativo entre las variables clínico-patológicas y moleculares según los subgrupos. CMS: Subtipo molecular Consenso; MSI: Inestabilidad microsatelital; CIMP: fenotipo metilador de islotes CpG; CIN: inestabilidad cromosomal; IMC: Índice de masa corporal; dMMR: deficiencia del sistema reparador "mismatch repair". $\square$ Positivo; $\square$ Negativo; $\square$ No determinado.

mayormente al lado izquierdo, mucinosos, sin invasión vascular ni linfática y sin mutaciones en BRAF. No se observaron metástasis sincrónicas. Tuvo el mayor porcentaje de KRAS ${ }^{\text {mut }}$ entre los grupos (Tabla 5, Figura 2).

Las características asociadas a tumores más agresivos como ausencia de mucina y bajo grado de diferenciación se observaron en los tumores CMS4, que correspondieron a $21 \%$. En este grupo el estado de CIMP-alta no se acompaña de MLH1 hipermetilado, por lo que son tumores MSS (Tabla 5 , Figura 2). En este grupo se observó una frecuencia de 20\% (2/10) de KRAS $^{\text {mut }}$ (Tabla 5).

Con respecto a mutaciones en PI3KCA no se observaron diferencias entre los grupos (Tabla 5).

\section{Discusión}

El presente estudio fue diseñado para caracterizar y describir las tres principales vías moleculares carcinogénicas (CIN, MSI y CIMP) involucradas en la heterogeneidad y complejidad molecular en el CCR.

En la práctica clínica, la clasificación $\mathrm{TNM}^{28,29}$ es utilizada como la principal herramienta pronóstica de selección de pacientes a quimioterapia adyuvante. Se ha descrito que la sobrevida de pacientes en etapas tempranas supera $90 \%$, pero en estadios metastásicos es inferior a $10 \%{ }^{30}$. En los últimos años, la incorporación de terapias moleculares ha permitido mejorar la sobrevida de estos pacientes, sin embargo, debido a la heterogeneidad de los tumores, no tienen la misma efectividad en todos los pacientes ${ }^{31-34}$.

Considerando lo anterior, el año 2015 se estableció un grupo internacional de subtipificación del CCR, The CRC Subtyping Consortium, basado en datos provenientes de The Cancer Genome Atlas complementados con análisis moleculares y mutacionales ${ }^{19}$. Se establecieron 4 subgrupos de consenso: CMS1 (MSI-inmune), CMS2 (canónica), CMS3 (metabólica), y CMS4 (mensenquimal) con una prevalencia en la población internacional de $14 \%, 37 \%, 13 \%$ y $23 \%$, respectivamente. Esta clasificación refleja una evolución biológica específica durante el desarrollo de los tumores, permitiendo definir terapias con mejor respuesta y sobrevida de los pacientes ${ }^{19,33,35}$.

En este estudio se logró subclasificar a los pacientes chilenos participantes en los 4 subgrupos establecidos por el CRCSC y la prevalencia para cada subgrupo de $19 \%, 31 \%$, 29\% y $21 \%$ para CMS1, CMS2, CMS3 y CMS4 respectivamente, es acorde a lo descrito ${ }^{19,32,33}$ (Tabla 6).

Se describió que los tumores CMS1 presentan 
Tabla 6. Resumen de la clasificación de los tumores de CCR en 4 subtipos basados en las variables clínico-patológicas y moleculares

\begin{tabular}{|lcccc|}
\hline $\mathbf{n}=\mathbf{4 8}$ & CMS1 & CMS2 & CMS3 & CMS4 \\
$\%$ & 19 & 31 & 29 & 21 \\
$\mathrm{MSI}$ & +++ & - & $+/-$ & - \\
CIMP & + & - & - & +++ \\
CIN & - & +++ & - & + \\
KRAS & - & + & + & + \\
BRAF & + & - & - & - \\
PI3KCA & + & + & + & + \\
Localización & $\mathrm{D}>\mathrm{I}$ & $\mathrm{I}>\mathrm{D}$ & $\mathrm{I}>\mathrm{D}$ & $\mathrm{D}>\mathrm{I}$ \\
Género & $\mathrm{F}>\mathrm{M}$ & $\mathrm{M}>\mathrm{F}$ & $\mathrm{M}>\mathrm{F}$ & $\mathrm{F}>\mathrm{M}$ \\
\hline Mucina & +++ & + & + & - \\
\hline Diferenciación & + & +++ & +++ & ++ \\
\hline
\end{tabular}

D: derecho; I: izquierdo; F: femenino; M: masculino; MSI: inestabilidad microsatelital; CIMP: fenotipo metilador de islotes CpG; CIN: inestabilidad cromosomal; CMS: subtipo molecular consenso.

características que previamente se han reportado para tumores MSI-alta/CIMP-alta en CCR esporádico ${ }^{15}$. Se ha descrito como lesión precursora a los pólipos sésiles aserrados ${ }^{36}$. Estos tumores presentan una alta respuesta de infiltrado intratumoral, lo cual confiere a estos pacientes un buen pronóstico y, según lo reportado, se sugiere que ello se debería a la presencia de linfocitos T citotóxicos $\mathrm{CD} 8+{ }^{37-40}$. Por otra parte, se ha establecido que la mutación activante en el oncogen BRAF (V600E) provoca la activación constitutiva de la vía EGFR $y$ en consecuencia una ineficiente respuesta al tratamiento con cetuximab o panitumumab. Cabe señalar que estos pacientes poseen un pronóstico intermedio $^{33}$ y una tasa de supervivencia muy pobre después de la recaída ${ }^{38}$. Debido a la falta de datos de seguimiento no fue posible establecer esta correlación.

Se ha reportado que los tumores CMS2 se originan a partir de adenomas tubulares, cuya progresión adenoma-carcinoma es iniciada por el gen supresor de tumores APC con una alta actividad en la vía de señalización intracelular Wnt/MYC ${ }^{35,41}$. Si bien no contamos con perfiles moleculares que den cuenta de la activación de la vía Wnt/MYC, logramos establecer que los tumores categorizados CMS2 se asocian a mutaciones en oncogenes como KRAS y PI3KCA y poseen características clínicas asociadas a una mejor respuesta a terapias asociadas a la vía CIN-alta. Se ha descrito una mejor sobrevida después de la recurrencia comparado a otros subtipos ${ }^{35}$.

Hemos observado que tumores de pacientes categorizados en CMS3 exhiben la mayor tasa de mutación activante en el oncogen KRAS, indicando que estos pacientes tendrían una baja respuesta al tratamiento con cetuximab o panitumumab. Se ha descrito que este subgrupo corresponde a tumores epiteliales de pronóstico intermedio y cuya lesión precursora aún no se ha determinado ${ }^{33}$.

Los tumores CMS4 se han descrito como el subtipo de peor pronóstico ${ }^{19,33}$. Estos tumores provienen de pólipos sésiles aserrados, pero a diferencia del subtipo CMS1, presentan sobreexpresión de señales asociadas a la activación del factor de crecimiento transformante (TGF- $\beta$ ) que desencadena señales intracelulares asociadas a la transición epitelio-mesénquima (MET) ${ }^{35,41}$.

Según nuestros datos, al momento del diagnóstico, la mayor prevalencia de tumores en estados avanzados fueron categorizados en CMS2 y CMS4. Además, presentaron la mayor proporción de metástasis (sincrónica y metacrónica). Estos resultados se podrían explicar por el alto número de ganancia o pérdida de copias de ongenes o genes supresores de tumores presente en ambos grupos. Sin embargo, el peor pronóstico asociado al grupo CMS4 podría explicarse, además de la activación de TGF- $\beta$, por remodelamiento de la matriz, angiogénesis, invasión estromal y por alto estado de metilación de genes supresores de tumores que caracteriza a este subtipo.

En resumen, hemos logrado distinguir 4 subgrupos en CCR, con señales intracelulares diferentes que le confieren una biología única a cada tumor. Si bien esta subclasificación se ha asociado a pronóstico y respuesta a terapia, en nuestra serie no fue posible de establecer debido al corto período de seguimiento y al tamaño de nuestro grupo de estudio, siendo una de nuestras limitaciones. Se espera que la clasificación de consenso en estos 4 subgrupos faciliten la incorporación de las nuevas terapias establecidas en los grandes grupos de investigación, basadas en la heterogeneidad del tumor para dar terapia más efectiva a cada paciente. 


\section{Referencias}

1. Ferlay J, Soerjomataram I, Dikshit R, Eser S, Mathers $\mathrm{C}$, Rebelo $\mathrm{M}$, et al. Cancer incidence and mortality wordwild: source, methods and major patterns in GLOBOCAN 2012. Int J Cancer 2015; 136 E359-86.

2. Bray F, Ferlay J, Laversanne M, Brewster DH, Gombe Mbalawa C, Kohler B, et al. Cancer Incidence in Five Continents: Inclusion criteria, highlights from Volume $\mathrm{X}$ and the global status of cancer registration. Int J Cancer 2015; 137 (9): 2060-71.

3. Zárate AJ, Alonso FT, Garmendia ML, López-Köstner F. Increasing crude and adjusted mortality rates for colorectal cancer in a developing South American country. Colorectal Dis 2013; 15 (1): 47-51.

4. Vogelstein B, Fearon ER, Hamilton SR, Kern SE, Preisinger AC, Leppert M, et al. Genetic alterations during colorectal-tumor development. N Engl J Med 1988; 319 (9): 525-32.

5. Vogelstein B, Kinzler KW. The Path to Cancer-Three Strikes and You're Out. N Engl J Med 2015; 373.

6. Yiu AJ, Yiu CY. Biomarkers in Colorectal Cancer. Anticancer Research 2016; 36: 1093-102.

7. Colussi D, Brandi G, Bazzoli F, Ricciardiello LC. Molecular Pathways Involved in Colorectal Cancer: Implications for disease behavior and prevention. Int J Mol Sci 2013; 14: 16365-85.

8. Kudryavtseva AV, Lipatova AV, Zaretsky AR, Moskalev AA, Fedorova MS, Rasskazova AS, et al. Important molecular genetic markers of colorectal cancer Oncotarget 2016 Jun 2. doi: 10.18632/oncotarget.9796.

9. Watanabe T, Kobunai T, Yamamoto Y, Matsuda K, Ishihara S, Nozawa K, et al. Chromosomal instability (CIN) phenotype, CIN high or CIN low, predicts survival for colorectal cancer. J Clin Oncol 2012; 30 (18): 2256-64.

10. Hveem TS, Merok MA, Pretorius ME, Novelli M, Bævre MS, Sjo OH, et al. Prognostic impact of genomic instability in colorectal cancer BJC 2014; 110: 2159-64.

11. Mouradov D, Domingo E, Gibbs P, Jorissen RN, Li S, Soo PY, et al. Survival in stage II/III colorectal cancer is independently predicted by chromosomal and microsatellite instability, but not by specific driver mutations Am J Gastroenterol 2013; 108 (11): 1785-93.

12. Issa JP. Colon cancer: it's CIN or CIMP. Clin Cancer Res 2008; 14 (19): 5939-40.

13. Zhang X, Shimodaira H, Soeda H, Komine K, Takahashi $\mathrm{H}$, Ouchi $\mathrm{K}$, et al. CpG island methylator phenotype is associated with the efficacy of sequential oxaliplatinand irinotecan-based chemotherapy and EGFR-related gene mutation in Japanese patients with metastatic colorectal cancer. Int J Clin Oncol 2016; 21 (6): 1091-101.
14. Cha Y, Kim KJ, Han SW, Rhee YY, Bae JM, Wen X, et al. Adverse prognostic impact of the $\mathrm{CpG}$ island methylator phenotype in metastatic colorectal cancer. Br J Cancer 2016; 115 (2): 164-71.

15. Jass JR. Classification of colorectal cancer based on correlation of clinical, morphological and molecular features. Histopathology 2007; 50: 113-30.

16. Sadanandam A, Wang X, de Sousa E Melo F, Gray JW, Vermeulen L, et al. Reconciliation of classification systems defining molecular subtypes of colorectal cáncer: Interrelationships and clinical implications. Cell Cycle 2014; 13 (3): 353-7.

17. Marisa L, de Reyniès A, Duval A, Selves J, Gaub MP, Vescovo L, et al. Gene expression classification of colon cancer into molecular subtypes: characterization, validation, and prognostic value. PLoS Med 2013; 10 (5): e1001453.

18. Domingo E, Ramamoorthy R, Oukrif D, Rosmarin D, Presz $\mathrm{M}$, Wang $\mathrm{H}$, et al. Use of multivariate analysis to suggest a new molecular classification of colorectal cancer. J Pathol 2013; 229 (3): 441-8.

19. Guinney J, Dienstmann R, Wang X, de Reyniès A, Schlicker A, Soneson C, et al. The consensus molecular subtypes of colorectal cancer. Nat Med 2015; 21 (11): 1350-6.

20. Lahiri DK, Nurnberger JI Jr. A rapid non-enzymatic method for the preparation of HMW DNA from blood for RFLP studies. Nucleic Acids Res 1991; 19: 5444.

21. Cawkwell L1, Lewis FA, Quirke P. Frequency of allele loss of DCC, p53, RBI, WT1, NF1, NM23 and APC/ MCC in colorectal cancer assayed by fluorescent multiplex polymerase chain reaction. Br J Cancer 1994; 70 (5): 813-8.

22. Chang SC, Lin JK, Lin TC, Liang WY. Loss of heterozygosity: an independent prognostic factor of colorectal cancer. World J Gastroenterol 2005; 11 (6): 778-84.

23. Wielandt AM, Zárate AJ, Hurtado C, Orellana P, Álvarez K, Pinto E, et al. [Lynch syndrome: selection of families by microsatellite instability and immunohistochemistry]. Rev Med Chile 2012; 140 (9): 1132-9.

24. Eads CA, Danenberg KD, Kawakami K, Saltz LB, Blake C, Shibata D, et al. Methy Light: a high-throughput assay to measure DNA methylation. Nucleic Acids Res 2000; 28 (8): E32.

25. Berg M, Hagland HR, Søreide K. Comparison of CpG island methylator phenotype (CIMP) frequency in colon cancer using different probe- and gene-specific scoring alternatives on recommended multi-gene panels. PLoS One 2014; 9 (1): e86657.

26. Weisenberger DJ, Siegmund KD, Campan M, Young J, Long TI, Faasse MA, et al. CpG island methylator phe- 
notype underlies sporadic microsatellite instability and is tightly associated with BRAF mutation in colorectal cancer. Nat Genet 2006; 38 (7): 787-93.

27. Hurtado C, Wielandt AM, Zárate AJ, Kronberg U, Castro M, Yamagiwa K, et al. [Molecular analysis of sporadic colon cáncer]. Rev Med Chile 2015; 143 (3): 310-9.

28. Edge SB, Byrd DR, Compton CC, Fritz AG, Greene FL, Trotti A. AJCC Cancer Staging Manual. 7th ed. NewYork: Springer; 2010.

29. Greene FL. Current TNM staging of colorectal cancer. Lancet Oncol 2007; 8 (7): 572-3.

30. Hardy RG, Meltzar SJ, Jankowski JA, ABC of Colorectal Cancer: Molecular basis for risk factors BJM 2000; 321: 886-9.

31. Zlobec I, Kovac M, Erzberger P, Molinari F, Bihl MP, Rufle A, et al. Combined analysis of specific KRAS mutation, BRAF and microsatellite instability identifies prognostic subgroups of sporadic and hereditary colorectal cancer. Int J Cancer 2010; 127 (11): 2569-75.

32. Fessler E, Jansen M, De Sousa F, Melo E, Zhao L, Prasetyanti PR, et al. A multidimensional network approach reveals microRNAs as determinants of the mesenchymal colorectal cancer subtype. Oncogene 2016; 35: 6026-37.

33. Punt C, Koopman M, Vermeulen L. From tumour heterogeneity to advances in precision treatment of colorectal cancer. Nat Rev Clin Oncol 2017;14 (4): 235-46.

34. Blanco-Calvo M, Concha Á Figueroa A, Garrido F, Valladares-Ayerbes M. Colorectal Cancer Classification and Cell Heterogeneity: A Systems Oncology Approach. Int J Mol Sci 2015; 16 (6): 13610-32.
35. Müller MF, Ibrahim AE, Arends MJ. Molecular pathological classification of colorectal cancer. Virchows Arch 2016; 469 (2): 125-34.

36. De Sousa E, Wang X, Jansen M, Fessler E, Trinh A, de Rooij LP, et al. Poor-prognosis colon cancer is defined by a molecularly distinct subtype and develops from serrated precursor lesions. Nat Med 2013; 19 (5): 6148.

37. Zárate AJ, Álvarez K, Villarroel C, Wielandt AM, Kronberg U, Cavada G, et al. Caracterización del infiltrado linfocitario ( $\mathrm{cd} 3, \mathrm{~cd} 4, \mathrm{~cd} 8, \mathrm{~cd} 45$ ro y foxp3) e inestabilidad microsatelital en pacientes con cáncer colorrectal. Rev Chil Cir 2015; 67 (1): 43-50.

38. Dunne PD, O’Reilly PG, Coleman HG, Gray RT, Longley DB, Johnston PG, et al. Stratified analysis reveals chemokine-like factor (CKLF) as a potential prognostic marker in the MSI-immune consensus molecular subtype CMS1 of colorectal cancer. Oncotarget 2016; 7 (24): 36632-44.

39. Le DT, Uram JN, Wang H, Bartlett BR, Kemberling $\mathrm{H}$, Eyring $\mathrm{AD}$, et al. PD-1 Blockade in Tumors with Mismatch-Repair Deficiency N Engl J Med 2015; 372: 2509-20.

40. Becht E, de Reyniès A, Giraldo NA, Pilati C, Buttard B, Lacroix L, et al. Immune and Stromal Classification of Colorectal Cancer Is Associated with Molecular Subtypes and Relevant for Precision Immunotherapy. Clin Cancer Res 2016; 22 (16): 4057-66.

41. Fessler E, Drost J, van Hooff SR, Linnekamp JF, Wang $\mathrm{X}$, Jansen $\mathrm{M}$, et al. TGF signaling directs serrated adenomas to the mesenchymal colorectal cancer subtype. EMBO Mol Med 2016; 8 (7): 745-60. 